Pacific Journal of Mathematics

BOREL DENSITY, THE MARGINAL PROBLEM AND
ISOMORPHISM TYPES OF 


\title{
BOREL DENSITY, THE MARGINAL PROBLEM AND ISOMORPHISM TYPES OF ANALYTIC SETS
}

\author{
R. M. SHORTT
}

The present work arises out of an ongoing study of the existence of probabilities with prescribed marginals, in particular, an attempt to determine exactly for which spaces property (V) and the Kolmogoroff consistency theorem hold. To this end, we have introduced the concept of Borel-density, in fact an infinite hierarchy of Borel-densities (see Proposition 3). Their relationship to the marginal problem is explored in Propositions 9 and 10: density of order 3 implies property (V) and, in the presence of order 2 density, is equivalent with it. Propositions 11 and 12 treat Kolmogoroff consistency problems: infinite-order Borel-density is sufficient for Kolmogoroff's theorem to hold; as a consequence, there are highly non-measurable spaces over which the theorem obtains. Finally, and perhaps most intriguingly, there are applications of these results to the (open) problem of determining the isomorphism types of analytic sets. Proposition 13 asserts that if $X_{1}$ and $X_{2}$ are uncountable separable spaces such that $X_{1} \times X_{2}$ is Borel-dense, then $X_{1}$ and $X_{2}$ are standard.

This last result improves a theorem of R. D. Mauldin (1976) to the effect that if an analytic (non-Borel) subset $A$ of the unit interval has totally imperfect complement, then $A$ is not isomorphic with $A^{n}, n \geq 2$. A consequence of our Proposition 13 (Corollary 7) is that such an $A$ is not isomorphic with any product $B \times C$ of uncountable spaces $B$ and $C$. We do not use the method of Lusin sieves.

The definition of $n$th order Borel-density bears some formal resemblance to certain work of Cox (1980) on Lusin properties for Cartesian products, but the exact link seems unclear.

Notation and terminology. We work exclusively with separable spaces, i.e. measurable spaces $(X, \mathscr{B})$ with a countably generated and separated $\sigma$-algebra $\mathscr{B}$. Often, the notation of a $\sigma$-algebra is suppressed: the space is called by $X$ alone and, when needed, its measurable structure is indicated by $\Re=\Re(X)$. If $A$ is a subset of $X$, consider $A$ as another separable space with $\Re(A)=\{A \cap B: B \in \Re\}$.

A separable space $(X, \mathscr{B})$ is standard if there is a separable, topologically complete metric $d$ on $X$ such that $\Re$ is the Borel $\sigma$-algebra of the metric space $(X, d)$. A metric space is standard if its Borel structure is standard. If $X$ is a separable metric space with Borel $\sigma$-algebra $\mathscr{B}$, then $(X, \mathscr{B})$ is a separable space. Furthermore, there is a well-known result due to Marczewski (1938) stating that if $(X, \mathscr{B})$ is a separable space, then 
there is a separable metric $d$ on $X$ such that $\mathscr{B}$ is the Borel $\sigma$-algebra of the metric space $(X, d)$. It follows from this that every separable space is a subset of some standard space.

We use the terms "probability measure" and "law" interchangeably; we denote the inner and outer measures formed from a law $P$ by $P_{*}$ and $P^{*}$, respectively. If $A \subset X$, and $Q$ is a law on $(A, \Re(A))$, we define the law $\bar{Q}$ induced by $Q$ on $X$ by the rule $\bar{Q}(B)=Q(B \cap A)$, for $B \in \mathscr{B}(X)$. It is easy to verify that:

(1) $P^{*}$ is a finite measure on $(A, \Re(A))$,

(2) $(\bar{Q})^{*}=Q$ on $\Re(A)$, and

(3) if $P^{*}(A)=1$, then $\overline{P^{*}}=P$.

A separable metric space $(X, d)$ is universally measurable $(u . m$.$) if for$ every law $P$ on its completion $\bar{X}$, there are Borel subsets $A$ and $B$ of $\bar{X}$ such that $A \subset X \subset B$ and $P(A)=P(B)$. The following result is most useful:

LEMMA 1. Let $X$ be $a$ set and let $d_{1}$ and $d_{2}$ be separable metrics generating the same Borel structure; then $\left(X, d_{1}\right)$ is u.m. if and only if $\left(X, d_{2}\right)$ is u.m. (See Shortt [15].)

We are thus justified in calling a separable space $X$ u.m. if it is Borel-isomorphic with some u.m. metric space.

A law $P$ on a separable space $X$ is continuous if $P\{x\}=0$ for each $x \in X$. A separable $X$ is universally null if there are no continuous laws on $(X, \Re(X))$.

A separable space $X$ is analytic if it is the measurable image of a standard space; $X$ is co-analytic if it is Borel-isomorphic with the complement of an analytic subset of a standard space. It is well-known that analytic and co-analytic spaces are u.m.

In much of the following enterprise, we shall be considering a sequence (possibly infinite) of (usually uncountable standard) spaces $S_{1}, S_{2}, \ldots, S_{n}$ and various subsets $A$ of $S_{1} \times S_{2} \times \cdots \times S_{n}$. By a $k$-slice of $S_{1} \times \cdots \times S_{n}$ we mean a set of the form $A_{1} \times \cdots \times A_{n}$, where $k$ of these factor sets are singletons and the other $n-k$ sets $A_{j}$ are equal to $S_{j}$ (i.e. $k$ co-ordinates have been specified; $1 \leq k \leq n$ ). If $\left\{i_{1}, \ldots, i_{k}\right\}$ is a subset of $\{1, \ldots, n\}$ of cardinality $k$, and $s_{1}, \ldots, s_{k}$ are elements of $S_{i_{1}}, \ldots, S_{i_{k}}$, respectively, then $\left\{\vec{s} \in S_{1} \times \cdots \times S_{n}: \vec{s}\left(i_{j}\right)=s_{j}, j=1, \ldots, k\right\}$ is the $k$-slice of $S_{1} \times \cdots \times S_{n}$ over the point $\left(s_{1}, \ldots, s_{k}\right)$; it will occasionally be identified with its projection on $\Pi\left\{S_{i}: i \neq i_{j}, j=1, \ldots, k\right\}$. 
If $A \subset S_{1} \times \cdots \times S_{n}$, then by a $k$-section of $A$ we mean the intersection of $A$ with a $k$-slice of $S_{1} \times \cdots \times S_{n}$; if $B$ is a $k$-slice of $S_{1} \times \cdots \times S_{n}$ over the point $\left(s_{1}, \ldots, s_{k}\right)$, then $B \cap A$ is the $k$-section of $A$ over the point $\left(s_{1}, \ldots, s_{k}\right)$. Again, these sets are sometimes identified with their projections on the corresponding $n-k$-dimensional partial product. Most often, $k=1$.

LEMMA 2. Let $E$ and $F$ be analytic spaces and let $A$ be an analytic subset of $E \times F$. If $A(y)=\{x \in E:(x, y) \in A\}$ denotes the 1-section of $A$ over the point $y$, then $\{y \in F: A(y)$ is uncountable $\}$ is an analytic subset of $F$.

Proof. This theorem is orginally due to Mazurkiewicz and Sierpinski (1924) and has been generalised by Kuratowski [7, p. 261], Saks [14, p. 218], and Hoffmann-Jørgensen [6, III.6.1].

LEMMA 3. Let $A$ be a standard subset of the product $E \times F$ of analytic spaces $E$ and $F$. If the 1-sections $A(x)=\{y \in F:(x, y) \in A\}$ are countable for all $x$ in $E$, then there exist standard sets $B_{n} \subset E(n=1,2, \ldots)$, and measurable mappings $f_{n}: B_{n} \rightarrow F$ such that:

(1) $f_{n}(x) \neq f_{m}(x)$ for all $x$ in $B_{n} \cap B_{m}$ and $n \neq m$, and

(2) $A=\cup_{n=1}^{\infty} G\left(f_{n}\right)$, where $G\left(f_{n}\right)$ is the graph of $f_{n}$.

Proof. This theorem is essentially due to Lusin [8, p. 243]; a proof is to be found in Hahn [5] or Hoffmann-Jørgensen [6, III.6.7].

Let $E$ and $F$ be separable spaces and let $S$ be an uncountable standard subset of $E \times F$. Given $x_{0}$ in $E$ and $y_{0}$ in $F$, define the 1-sections

$$
\begin{aligned}
& S_{1}\left(x_{0}\right)=\left\{y \in F:\left(x_{0}, y\right) \in S\right\} \quad \text { and } \\
& S_{2}\left(y_{0}\right)=\left\{x \in E:\left(x, y_{0}\right) \in S\right\} .
\end{aligned}
$$

LeMma 4. Suppose that for each $x \in E$ and $y \in F$, one has $S_{1}(x)$ and $S_{2}(y)$ countable; then there is an uncountable standard subset $S_{0}$ of $E$ and a one-one measurable function $f: S_{0} \rightarrow F$ whose graph $G(f)$ is contained in $S$.

Proof. Using Lemma 3, we find (for $n=1,2, \ldots$ ) standard subsets $B_{n} \subset E$ and measurable mappings $f_{n}: B_{n} \rightarrow F$ so that $S=\cup_{n=1}^{\infty} G\left(f_{n}\right)$; select $n$ so that $G\left(f_{n}\right)$ is uncountable. Notice that since $g_{n}: B_{n} \rightarrow S$ defined by $g_{n}(x)=\left(x, f_{n}(x)\right)$ is one-one and measurable, its range $G\left(f_{n}\right)$ belongs in $\mathscr{B}(S)$ and so is standard. 
Apply Lemma 3 once more, this time to the set $G\left(f_{n}\right)$, using the fact that its "horizontal" sections are countable. There are, for $m=1,2, \ldots$, standard subsets $C_{m} \subset F$ and measurable mappings $g_{m}: C_{m} \rightarrow E$ so that $G\left(f_{n}\right)=\cup_{m=1}^{\infty} G\left(g_{m}\right)$; select $m$ so that $G\left(g_{m}\right)$ is uncountable.

Since each "vertical" section of $G\left(f_{n}\right)$, hence of $G\left(g_{m}\right)$ is a singleton, $g_{m}: C_{m} \rightarrow B_{n}$ is one-one and so bimeasurable. We may put $S_{0}=g_{m}\left(C_{m}\right)$ and $f=g_{m}^{-1}$ on $S_{0}$.

The same argument shows that $S$ is the countable union of such graphs.

Before commencing the main section of this paper, we remark that although transfinite induction is in frequent employ throughout what follows, every attempt has been made to avoid the use of the Continuum Hypothesis $(\mathrm{CH})$. Wherever $\mathrm{CH}$ of its weakening Martin's Axiom is used, we have so noted it.

Borel-density. Let $E$ be a separable space; a subset $X$ of $E$ is Borel-dense in $E$ if whenever $B \in \Re(E)$ and $B \subset E \backslash X$, then $B$ is of cardinality less than $c$. For general $E$, this seems not to be a tame concept: for example, if $X$ is Borel-dense in $Y$, and $Y$ is Borel-dense in $Z$, can one conclude, without assumption of the Continuum Hypothesis, that $X$ is Borel-dense in $Z$ ? For our purposes, however, only the case where $X$ is Borel-dense in a standard space is of relevance.

Say that a separable space $X$ is Borel-dense if there is some standard space $S$ such that $S \backslash X$ contains no uncountable members of $\Re(S)$, equivalently, upon any metrisation, $S \backslash X$ contains no homeomorph of the Cantor discontinuum (the term "totally imperfect" is used to indicate such a set $S \backslash X)$. The term "density" is, I believe, quite appropriate: if $X$ is Borel-dense in the standard space $S$, and $f$ and $g$ are real measurable functions on $S$ agreeing on $X$, then $\{x: f(x) \neq g(x)\}$ is only countable.

Proposition 1. If $X$ is Borel-dense, then $X$ is of cardinality $c$.

Demonstration. Every uncountable standard space $S$ is the disjoint union of continuum many uncountable standard sets. (For example, if $S$ is uncountable standard, then $S \cong S \times S=\bigcup\{\{s\} \times S: s \in S\}$.) If now $X$ is Borel-dense in $S, X$ must meet each of these sets.

Let $S_{1}, S_{2}, S_{3}, \ldots$ be uncountable standard metric spaces and let $X_{1} \subset S_{1}, X_{2} \subset S_{2}, \ldots$ be subsets of these. For each $n=1,2, \ldots$, consider the following twelve propositions: 
$\left(\mathrm{K}_{n}\right)$ If $K$ is a compact subset of $\left(S_{1} \times \cdots \times S_{n}\right) \backslash\left(X_{1} \times \cdots \times X_{n}\right)$, then $K$ is contained in the union of fewer than $c$ 1-slices of $S_{1} \times \cdots \times S_{n}$ over points in $S_{j} \backslash X_{j}, j=1,2, \ldots, n$.

$\left(\mathrm{KK}_{n}\right)$ If $K$ is a compact subset of $\left(S_{1} \times \cdots \times S_{n}\right) \backslash\left(X_{1} \times \cdots \times X_{n}\right)$, then $K$ is contained in the union of fewer than $c$ 1-slices of $S_{1} \times \cdots \times S_{n}$.

$\left(\mathrm{K}_{n}^{\prime}\right)$ If $K$ is a compact subset of $\left(S_{1} \times \cdots \times S_{n}\right) \backslash\left(X_{1} \times \cdots \times X_{n}\right)$, then $K$ is contained in a countable union of 1-slices of $S_{1} \times \cdots \times S_{n}$ over points in $S_{j} \backslash X_{j}, j=1, \ldots, n$.

$\left(\mathrm{KK}_{n}^{\prime}\right)$ If $K$ is a compact subset of $\left(S_{1} \times \cdots \times S_{n}\right) \backslash\left(X_{1} \times \cdots \times X_{n}\right)$, then $K$ is contained in a countable union of 1-slices of $S_{1} \times \cdots \times S_{n}$.

$\left(\mathrm{B}_{n}\right)$ If $B \in \Re_{(}\left(S_{1} \times \cdots \times S_{n}\right)$ is a subset of

$$
\left(S_{1} \times \cdots \times S_{n}\right) \backslash\left(X_{1} \times \cdots \times X_{n}\right),
$$

then $B$ is contained in the union of fewer than $c$ 1-slices of $S_{1} \times \cdots \times S_{n}$ over points in $S_{j} \backslash X_{j}, j=1, \ldots, n$.

$\left(\mathrm{BB}_{n}\right)$ If $B \in \Re_{(}\left(S_{1} \times \cdots \times S_{n}\right)$ is a subset of

$$
\left(S_{1} \times \cdots \times S_{n}\right) \backslash\left(X_{1} \times \cdots \times X_{n}\right),
$$

then $B$ is contained in the union of fewer than $c$ 1-slices of $S_{1} \times \cdots \times S_{n}$.

$\left(\mathrm{B}_{n}^{\prime}\right)$ If $B \in \mathscr{B}\left(S_{1} \times \cdots \times S_{n}\right)$ is a subset of

$$
\left(S_{1} \times \cdots \times S_{n}\right) \backslash\left(X_{1} \times \cdots \times X_{n}\right),
$$

then $B$ is contained in a countable union of 1-slices of $S_{1} \times \cdots \times S_{n}$ over points in $S_{j} \backslash X_{j}, j=1, \ldots, n$.

$\left(\mathrm{BB}_{n}^{\prime}\right)$ If $B \in \Re_{B}\left(S_{1} \times \cdots \times S_{n}\right)$ is a subset of

$$
\left(S_{1} \times \cdots \times S_{n}\right) \backslash\left(X_{1} \times \cdots \times X_{n}\right),
$$

then $B$ is contained in a countable union of 1-slices of $S_{1} \times \cdots \times S_{n}$.

$\left(\mathrm{A}_{n}\right)$ If $A$ is an analytic subset of $\left(S_{1} \times \cdots \times S_{n}\right) \backslash\left(X_{1} \times \cdots \times X_{n}\right)$, then $A$ is contained in the union of fewer than $c$ 1-slices of $S_{1} \times \cdots \times S_{n}$ over points in $S_{j} \backslash X_{j}, j=1, \ldots, n$.

$\left(\mathrm{AA}_{n}\right)$ If $A$ is an analytic subset of $\left(S_{1} \times \cdots \times S_{n}\right) \backslash\left(X_{1} \times \cdots \times X_{n}\right)$, then $A$ is contained in the union of fewer than $c$ 1-slices of $S_{1} \times \cdots \times S_{n}$.

$\left(\mathrm{A}_{n}^{\prime}\right)$ If $A$ is an analytic subset of $\left(S_{1} \times \cdots \times S_{n}\right) \backslash\left(X_{1} \times \cdots \times X_{n}\right)$, then $A$ is contained in a countable union of 1-slices of $S_{1} \times \cdots \times S_{n}$ over points in $S_{j} \backslash X_{j}, j=1, \ldots, n$.

$\left(\mathrm{AA}_{n}^{\prime}\right)$ If $A$ is an analytic subset of $\left(S_{1} \times \cdots \times S_{n}\right) \backslash\left(X_{1} \times \cdots \times X_{n}\right)$, then $A$ is contained in a countable union of 1-slices of $S_{1} \times \cdots \times S_{n}$. 
The case of $n=\infty$ is also allowed, but is treated separately. We know immediately that for each $n$ (including $n=\infty$ ), the following three-dimensional lattice of implications obtains:

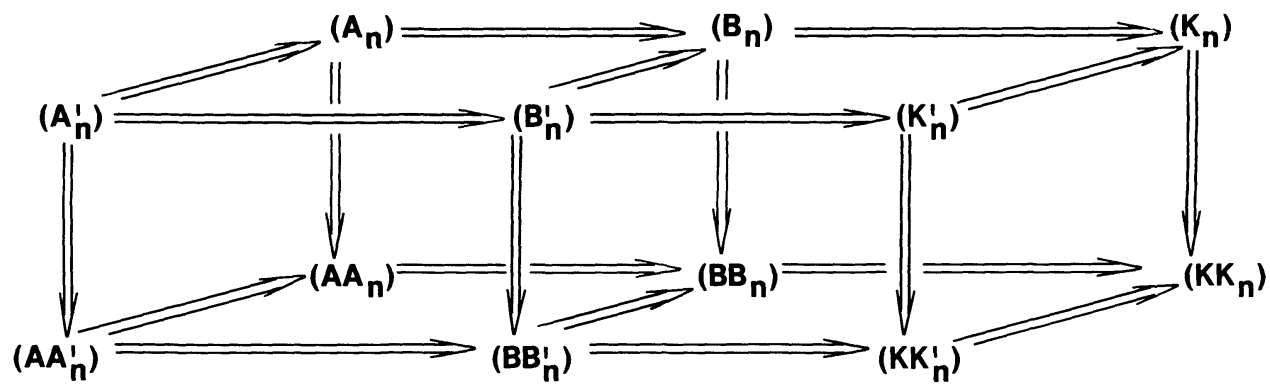

Additionally, for each $n \geq 2$, the following implications hold:

$(*)$

$$
\left\{\begin{aligned}
\left(\mathrm{K}_{\infty}\right) & \Rightarrow\left(\mathrm{K}_{n}\right) \Rightarrow\left(\mathrm{K}_{n-1}\right), \\
\left(\mathrm{KK}_{\infty}\right) & \Rightarrow\left(\mathrm{KK}_{n}\right) \Rightarrow\left(\mathrm{KK}_{n-1}\right), \\
\left(\mathrm{K}_{\infty}^{\prime}\right) & \Rightarrow\left(\mathrm{K}_{n}^{\prime}\right) \Rightarrow\left(\mathrm{K}_{n-1}^{\prime}\right), \\
\left(\mathrm{KK}_{\infty}^{\prime}\right) & \Rightarrow\left(\mathrm{KK}_{n}^{\prime}\right) \Rightarrow\left(\mathrm{KK}_{n-1}^{\prime}\right), \\
\left(\mathrm{B}_{\infty}\right) & \Rightarrow\left(\mathrm{B}_{n}\right) \Rightarrow\left(\mathrm{B}_{n-1}\right), \\
\left(\mathrm{BB}_{\infty}\right) & \Rightarrow\left(\mathrm{BB}_{n}\right) \Rightarrow\left(\mathrm{BB}_{n-1}\right), \\
\left(\mathrm{B}_{\infty}^{\prime}\right) & \Rightarrow\left(\mathrm{B}_{n}^{\prime}\right) \Rightarrow\left(\mathrm{B}_{n-1}^{\prime}\right), \\
\left(\mathrm{BB}_{\infty}^{\prime}\right) & \Rightarrow\left(\mathrm{BB}_{n}^{\prime}\right) \Rightarrow\left(\mathrm{BB}_{n-1}^{\prime}\right), \\
\left(\mathrm{A}_{\infty}\right) & \Rightarrow\left(\mathrm{A}_{n}\right) \Rightarrow\left(\mathrm{A}_{n-1}\right), \\
\left(\mathrm{AA}_{\infty}\right) & \Rightarrow\left(\mathrm{AA}_{n}\right) \Rightarrow\left(\mathrm{AA}_{n-1}\right), \\
\left(\mathrm{A}_{\infty}^{\prime}\right) & \Rightarrow\left(\mathrm{A}_{n}^{\prime}\right) \Rightarrow\left(\mathrm{A}_{n-1}^{\prime}\right), \\
\left(\mathrm{AA}_{\infty}^{\prime}\right) & \Rightarrow\left(\mathrm{AA}_{n}^{\prime}\right) \Rightarrow\left(\mathrm{AA}_{n-1}^{\prime}\right)
\end{aligned}\right.
$$

For example, if $K$ is a compact subset of

$$
\left(S_{1} \times \cdots \times S_{n-1}\right) \backslash\left(X_{1} \times \cdots \times X_{n-1}\right)
$$

not contained in fewer than $c$ [resp. countably many] 1-slices of $S_{1}$ $\times \cdots \times S_{n-1}$, then, letting $K^{\prime}$ be an uncountable compact subset of $S_{n}$, we see that $K \times K^{\prime}$ is a compact subset of

$$
\left(S_{1} \times \cdots \times S_{n}\right) \backslash\left(X_{1} \times \cdots \times X_{n}\right)
$$

not contained in the union of fewer than $c$ [resp. countably many] 1-slices of $S_{1} \times \cdots \times S_{n}$. 
Proposition 2. For each finite $n,\left(\mathrm{KK}_{n}\right) \Rightarrow\left(\mathrm{A}_{n}^{\prime}\right)$, so that the twelve conditions introduced are in fact equivalent.

REMARK. Because of this equivalence, we are justified in making this definition: if any one of these conditions holds, say that $X_{1}, \ldots, X_{n}$ are jointly Borel-dense (of order $n$ ) in $S_{1}, \ldots, S_{n}$.

Demonstration. The proof proceeds by induction on $n$. For $n=1$, all of these conditions collapse to our earlier notion of Borel-density. Suppose that the proposition obtains for all indices less than $n, n \geq 2$, and that $\left(\mathrm{KK}_{n}\right)$ holds for $X_{1}, \ldots, X_{n}$ in $S_{1}, \ldots, S_{n}$. For each positive $k<n$ and subset $\left\{i_{1}, \ldots, i_{k}\right\}$ of $\{1, \ldots, n\}$ of cardinality $k$, the spaces $X_{i_{1}}, \ldots, X_{i_{k}}$ are jointly Borel-dense (of order $k$ ) in $S_{l}, \ldots, S_{l_{k}}$ : this follows from the implications in $(*)$ and the induction hypothesis. Suppose that $A$ is an analytic subset of $\left(S_{1} \times \cdots \times S_{n}\right) \backslash\left(X_{1} \times \cdots \times X_{n}\right)$. We construct a sequence of analytic sets $A \supset A_{1} \supset A_{2} \supset \cdots \supset A_{n-1}$ as follows (again, the process is inductive):

(1) Select any subset $\left\{i_{1}, \ldots, i_{n-1}\right\}$ of $\{1, \ldots, n\}$ of cardinality $n-1$, and let $j$ be the element in $\{1, \ldots, n\} \backslash\left\{i_{1}, \ldots, i_{n-1}\right\}$. Consider the $(n-1)$ sections of $A$ over points of $S_{i_{1}} \times \cdots \times S_{l_{n-1}}$; in particular, over points in $X_{i_{1}} \times \cdots \times X_{i_{n-1}}$, these are analytic subsets of $S_{j} \backslash X_{j}$ and so are countable $\left(\mathrm{A}_{1}^{\prime}\right)$.

The set of all points in $S_{i_{1}} \times \cdots \times S_{i_{n-1}}$ for which these sections are uncountable is ( $\mathrm{v}$, Lemma 2$)$ an analytic subset of

$$
\left(S_{i_{1}} \times \cdots \times S_{i_{n-1}}\right) \backslash\left(X_{i_{1}} \times \cdots \times X_{i_{n-1}}\right)
$$

and so is contained $\left(\mathrm{A}_{n-1}^{\prime}\right)$ in a countable union of 1-slices of $S_{i_{1}}$ $\times \cdots \times S_{i_{n-1}}$ over points $p$ in $S_{l} \backslash X_{i}, i=i_{1}, i_{2}, \ldots, i_{n-1}$. Remove from $A$ the 1 -slices of $S_{1} \times \cdots \times S_{n}$ over these points $p$.

Repeat this procedure for each subset $\left\{i_{1}, \ldots, i_{n-1}\right\}$ of $\{1, \ldots, n\}$ of cardinality $n-1$. What remains of $A$ is an analytic set $A_{1}$ each of whose $(n-1)$-sections is countable.

(2) Suppose that $1<k<n$ and that $A_{k-1}$ has been defined so that

(a) $A_{k-1}$ is formed from $A$ by the removal of countably many 1-slices of $S_{1} \times \cdots \times S_{n}$ over points in $S_{j} \backslash X_{j}, j=1, \ldots, n$, and

(b) each $(n-k+1)$-section of $A_{k-1}$ is countable.

Select any subset $\left\{i_{1}, \ldots, i_{n-k}\right\}$ of $\{1, \ldots, n\}$ of cardinality $n-k$, and let $j_{1}, \ldots, j_{k}$ be the elements of $\{1, \ldots, n\} \backslash\left\{i_{1}, \ldots, i_{n-k}\right\}$. Consider the $(n-k)$-sections of $A_{k-1}$ over points of $S_{i_{1}} \times \cdots \times S_{i_{n-k}}$; in particular, over points in $X_{i_{1}} \times \cdots \times X_{i_{n-k}}$, these are analytic subsets of 
$\left(S_{j_{1}} \times \cdots \times S_{j_{k}}\right) \backslash\left(X_{j_{1}} \times \cdots \times X_{j_{k}}\right)$ with each 1-section countable (they are $(n-k)+1$-sections of $\left.A_{k-1}\right)$. But now, from $\left(\mathrm{A}_{\mathrm{k}}^{\prime}\right)$, these $(n-k)$-sections must be countable.

The set of all points in $S_{i_{1}} \times \cdots \times S_{i_{n-k}}$ for which these $(n-k)$ sections are uncountable is an analytic subset of

$$
\left(S_{i_{1}} \times \cdots \times S_{i_{n-k}}\right) \backslash\left(X_{i_{1}} \times \cdots \times X_{i_{n-k}}\right)
$$

and so is contained $\left(A_{n-k}^{\prime}\right)$ in a countable union of 1 -slices of $S_{i_{1}}$ $\times \cdots \times S_{i_{n-k}}$ over points $p$ in $S_{i} \backslash X_{i}, i=i_{1}, \ldots, i_{n-k}$. Remove from $A_{k-1}$ the 1-slices of $S_{1} \times \cdots \times S_{n}$ over these points $p$.

Repeat this procedure for each of the $\left(\begin{array}{c}n \\ n-k\end{array}\right)$ subsets $\left\{i_{1}, \ldots, i_{n-k}\right\}$ of $\{1, \ldots, n\}$ of cardinality $n-k$. What remains of $A_{k-1}$ is an analytic set $A_{k}$ such that:

(a) $A_{k}$ is formed from $A$ by the removal of countably many 1-slices of $S_{1} \times \cdots \times S_{n}$ over points in $S_{j} \backslash X_{j}, j=1, \ldots, n$, and

(b) each ( $n-k)$-section of $A_{k}$ is countable.

(3) Finally, we obtain the set $A_{n-1}$. If this set were uncountable, there is an uncountable compact $K$ with $K \subset A_{n-1}$. From $\left(\mathrm{KK}_{n}\right)$, this $K$ is contained in the union of fewer than $c$ 1-slices of $S_{1} \times \cdots \times S_{n}$; this is a contradiction. Therefore $A_{n-1}$ is countable, and $\left(\mathrm{A}_{n}^{\prime}\right)$ holds true.

I do not know whether Proposition 2 is true at $n=\infty$.

Let $S_{1}, \ldots, S_{n}$ be uncountable standard spaces and suppose that $X_{1} \subset S_{1}, \ldots, X_{n} \subset S_{n}$ are such that for each subset $\left\{i_{1}, \ldots, i_{k}\right\}$ of $\{1, \ldots, n\}$ of cardinality $k$, the $X_{i_{1}}, \ldots, X_{i_{k}}$ are jointly Borel-dense in $S_{i_{1}}, \ldots, S_{i_{k}}$; then we say that $X_{1}, \ldots, X_{n}$ are jointly Borel-dense of order $k$ in $S_{1}, \ldots, S_{n}$.

CoROllaRY 1. Suppose that $X_{1}, \ldots, X_{n}(n \geq 2)$ are jointly Boreldense of order $n-1$ in $S_{1}, \ldots, S_{n}$. If $A$ is an analytic subset of $\left(S_{1} \times \cdots \times S_{n}\right) \backslash\left(X_{1} \times \cdots \times X_{n}\right)$, then after removing countably many 1-sections of $A$ over points in $S_{j} \backslash X_{j}, j=1, \ldots, n$, the remaining set has all of its 1-sections countable.

Proof. Exactly the method used for the preceding proposition.

Corollary 2. Suppose that $X_{1}, \ldots, X_{n}(n \geq 2)$ are jointly Borel-dense of order $n-1$, but not of order $n$, in $S_{1}, \ldots, S_{n}$. Then there is an uncountable $B \in \Re\left(S_{1} \times \cdots \times S_{n}\right), \quad B \subset\left(S_{1} \times \cdots \times S_{n}\right) \backslash\left(X_{1} \times \cdots \times X_{n}\right)$ each of whose non-empty 1-sections is a singleton set (this cannot occur, of course, in the presence of nth order density). Thus, for each subset $\left\{i_{1}, \ldots, i_{k}\right\}$ of 
$\{1, \ldots, n\}$ of cardinality $k, 0<k<n, B$ is the graph of a measurable isomorphism of some Borel subset of $S_{i_{1}} \times \cdots \times S_{i_{k}}$ onto a Borel subset of $S_{j_{1}} \times \cdots \times S_{j_{n-k}}$, where $j_{1}, \ldots, j_{n-k}$ are the elements of $\{1, \ldots, n\} \backslash\left\{i_{1}, \ldots, i_{k}\right\}$.

Proof. Let $B \in \Re\left(S_{1} \times \cdots \times S_{n}\right)$ be a subset of

$$
\left(S_{1} \times \cdots \times S_{n}\right) \backslash\left(X_{1} \times \cdots \times X_{n}\right)
$$

not contained in a countable union of 1-slices of $S_{1} \times \cdots \times S_{n}$. Applying Corollary 1 , we see that $B$ may be chosen so that each of its 1 -sections is countable.

Lemma 4 implies that there is an uncountable $B_{1} \subset B, B_{1} \in$ $\Re\left(S_{1} \times \cdots \times S_{n}\right)$ such that $B_{1}$ is the graph of a one-one measurable function from some member of $\mathscr{B}\left(S_{1}\right)$ onto some member of $\Re\left(S_{2} \times \cdots \times S_{n}\right)$. Repeating this argument, we obtain uncountable sets $B \supset B_{1} \supset B_{2} \supset \cdots \supset B_{n}$ in $\Re\left(S_{1} \times \cdots \times S_{n}\right)$ such that $B_{i}$ is the graph of a one-one measurable function from some uncountable member of $\Re\left(S_{i}\right)$ onto some member of

$$
\Re\left(S_{1} \times \cdots \times S_{i-1} \times S_{i+1} \times \cdots \times S_{n}\right) .
$$

Every non-empty 1-section of the set $B_{n}$ is a singleton set.

The hierarchy of Borel-density conditions which we have constructed does not collapse into a triviality; the following results ensure this.

Proposition 3. Let $S$ be an uncountable standard space; for each $n=1,2, \ldots$, there is a subset $X$ of $S$ such that $X \times X$ (n-times) is jointly Borel-dense of order $n$ in $S, \ldots, S$ (n-times), but $X, \ldots, X(n+1$-times) is not jointly Borel-dense of order $n+1$ in $S, \ldots, S$ (again $n+1$-times).

REMARK. It will follow as a consequence of Corollary 5 infra that none of the spaces $X$ is universally measurable.

Demonstration. We may take $S$ to be the unit interval ]0, 1[ under the usual Borel structure. Let $f_{1}, f_{2}, f_{3}, \ldots$ be the Borel automorphisms of $S$ defined by the rule

$$
f_{k}(s)=s^{k}
$$

Then

$$
f_{k}(s)=f_{l}(s) \text { for some } s \text { in } S \text { implies } k=l \text {. }
$$


Well-order the class of sets in $\mathscr{B}\left(S^{n}\right)$ not contained in the union of fewer than $c$ 1-slices of $S^{n}$ as $B_{0}, B_{1}, B_{2}, \ldots, B_{\alpha}, \ldots, \alpha<c$. Select any $n$-tuple $\left(x_{0}(1), \ldots, x_{0}(n)\right)$ in $B_{0}$ and in general for $\alpha<c$, take $\left(x_{\alpha}(1), \ldots, x_{\alpha}(n)\right)$ in $B_{\alpha} \backslash A_{\alpha}$, where $A_{\alpha}$ is the union of all 1-slices of $S^{n}$ over all points $f_{k}^{-1} f_{l}\left(x_{\beta}(j)\right)$ for all $j=1, \ldots, n, k=1,2, \ldots, n+1, l=1,2, \ldots, n+1$, all $\beta<\alpha$ (the 1-slices of $S^{n}$ over these points in each co-ordinate are removed; there are fewer than $c$ of these 1-slices).

Put $X=\left\{x_{\alpha}(j): j=1, \ldots, n, \alpha<c\right\}$. Then $X, \ldots, X$ ( $n$-times) is jointly Borel-dense of order $n$ in $S, \ldots, S$ ( $X^{n}$ meets each of the sets $\left.B_{\alpha}\right)$; however, we show now that $X^{n+1}$ does not intersect the set $G \in \mathscr{B}\left(S^{n+1}\right)$, where

$$
\begin{aligned}
& G=\{(s(1), \ldots, s(n), s(n+1)): \\
& \left.\quad f_{1}(s(1))=f_{2}(s(2))=\cdots=f_{n}(s(n))=f_{n+1}(s(n+1))\right\} .
\end{aligned}
$$

(Every 1-section of $G$ is a singleton, so that $G$ cannot be contained in the union of fewer than $c$ 1-slices of $S^{n+1}$.)

For the sake of argument, suppose that there is some element $(s(1), \ldots, s(n), s(n+1))$ in $X^{n+1} \cap G$. Condition $(*)$ for the $f_{k}$ implies that no two of these $s(j)$ are the same. Since at each stage of the construction of $X$, at most $n$ elements are chosen, there are (das Fachprinzip) ordinals $\beta<\alpha<c$ such that for some $i, j, k$, and $l, s(j)=x_{\alpha}(i)$, $s(k)=x_{\beta}(l)$ : this contradicts the choice of $\left(x_{\alpha}(1), \ldots, x_{\alpha}(n)\right)$, forcing it to lie in one of the forbidden 1-slices comprising $A_{\alpha}$.

Proposition 4. Let $S$ be an uncountable standard space; there is a subset $X$ of $S$ such that for each $n, X, \ldots, X$ (n-times) is jointly Borel-dense of order $n$ in $S, \ldots, S$, but $X, X, \ldots$ and $S, S, \ldots$ do not satisfy condition $\left(\mathrm{BB}_{\infty}\right)$.

Demonstration. Again, we take $S$ to be the interval $] 0,1[$; the functions $f_{1}, f_{2}, \ldots$ are as before.

Well-order the class of sets in $\Re(S) \cup \Re\left(S^{2}\right) \cup \Re\left(S^{3}\right) \cup \cdots$ not contained in the union of fewer than $c$ 1-slices (of $S, S^{2}, S^{3}, \ldots$, respectively) as $B_{0}, B_{1}, B_{2}, \ldots, B_{\alpha}, \ldots, \alpha<c$; for each ordinal $\alpha<c$, let $n=n(\alpha)$ be such that $B_{\alpha}$ is from $\Re\left(S^{n}\right)$. Select any $n(0)$-tuple $\left(x_{0}(1), \ldots, x_{0}(n(0))\right)$ in $B_{0}$ and in general for $\alpha<c$, take $\left(x_{\alpha}(1), \ldots, x_{\alpha}(n(\alpha))\right)$ in $B_{\alpha} \backslash A_{\alpha}$, where $A_{\alpha}$ is the union of all 1-slices of $S^{n(\alpha)}$ over all points $f_{k}^{-1} f_{l}\left(x_{\beta}(j)\right)$ for all $j=1, \ldots, n(\beta), k=1,2, \ldots, l=1,2, \ldots$, all $\beta<\alpha$ (the 1 -slices of $S^{n(\alpha)}$ over these points in each co-ordinate are removed; there are fewer than $c$ of these 1-slices). 
Put $X=\left\{x_{\alpha}(j): j=1, \ldots, n(\alpha), \alpha<c\right\}$. Then for each finite $n$, $X, \ldots, X$ ( $n$-times) is jointly Borel-dense of order $n$ in $S, \ldots, S$ ( $X^{n}$ meets each of the sets $B_{\alpha}$ such that $n=n(\alpha)$ ); however, we show now that $X^{\infty}$ does not intersect the set $G \in \mathscr{B}\left(S^{\infty}\right)$, where $G=\left\{(s(1), s(2), \ldots): f_{1}(s(1))\right.$ $\left.=f_{2}(s(2))=\cdots\right\}$. (As before, $G$ is not contained in the union of fewer than $c$ 1-slices of $S^{\infty}$.)

For the sake of argument, suppose that there is some element $(s(1), s(2), \ldots)$ in $X^{\infty} \cap G$. Condition (*) implies that no two of these $s(j)$ are the same. Since at each stage of the construction of $X$, only finitely many elements are chosen, these are ordinals $\beta<\alpha<c$ such that for some $i, j, k$, and $l, s(j)=x_{\alpha}(i), s(k)=x_{\beta}(l)$ : this contradicts the choice of $\left(x_{\alpha}(1), \ldots, x_{\alpha}(n(\alpha))\right)$, much as in Proposition 3.

Proposition 5. Let $S$ be an uncountable standard space; there is a subset $X$ of $S$ such that

(1) $X$ is not u.m., and yet

(2) the sequence $X, X, \ldots$ satisfies condition $\left(\mathrm{BB}_{\infty}\right)$ in $S, S, \ldots$

Demonstration. Well-order as $B_{0}, B_{1}, \ldots, B_{\alpha}, \ldots, \alpha<c$, all elements of $\Re\left(S^{\infty}\right)$ not contained in the union of fewer than $c$ 1-slices of $S^{\infty}$; also well-order as $C_{0}, C_{1}, \ldots, C_{\alpha}, \ldots, \alpha<c$, all uncountable members of $\Re(S)$.

Select $\left(x_{0}(1), x_{0}(2), \ldots\right)$ in $B_{0}$ and $w_{0}$ from $C_{0} \backslash\left\{x_{0}(j): j=1,2, \ldots\right\}$. In general, for any $\alpha<c$, choose $\left(x_{\alpha}(1), x_{\alpha}(2), \ldots\right)$ from $B_{\alpha} \backslash A_{\alpha}$, where $A_{\alpha}$ is the union of all 1-slices of $S^{\infty}$ (in every co-ordinate) over the points $\left\{w_{\beta}\right.$ : $\beta<\alpha\}$; choose $w_{\alpha}$ from $C_{\alpha} \backslash\left\{x_{\beta}(j): \beta \leq \alpha, j=1,2, \ldots\right\}$. Put $X=\left\{x_{\alpha}(j)\right.$ : $\alpha<c, j=1,2, \ldots\}$.

The $X, X, \ldots$ and $S, S, \ldots$ satisfy $\left(\mathrm{BB}_{\infty}\right)$, and yet both $X$ and its complement are Borel-dense in $S$, so that $X$ is not u.m.

\section{Relations with measure theory and the marginal problem.}

PROPOSITION 6. Let $S$ be an uncountable standard space; then $X \subset S$ is Borel-dense in $S$ if and only if $P^{*}(X)=1$ for every continuous law $P$ on $S$.

Proof. If $P^{*}(X)<1$, there is a (necessarily uncountable) set $B \in$ $\Re(S), X \cap B=\varnothing$ with $P(B)>0$.

Corollary 3. A set $X$ is u.m. and Borel-dense in $S$ if and only if $S \backslash X$ is universally null. 
Corollary 4. A co-analytic set $X$ is Borel-dense in $S$ if and only if $X$ is standard and $S \backslash X$ is countable. (Uncountable analytic sets are not universally null.)

Proposition 7. Let $S_{1}, \ldots, S_{n}$ be uncountable standard spaces and $X_{1} \subset S_{1}, \ldots, X_{n} \subset S_{n}$ be such that $X_{1}, \ldots, X_{n}$ are jointly Borel-dense (of order $n$ ) in $S_{1}, \ldots, S_{n}$; given laws $P_{1}, \ldots, P_{n}$ on $X_{1}, \ldots, X_{n}$, respectively, and $a$ law $Q$ on $S_{1} \times \cdots \times S_{n}$ with univariate marginals $\bar{P}_{1}, \ldots, \bar{P}_{n}$ (the laws induced by the $P_{j}$ on $\left.S_{j}, j=1, \ldots, n\right)$, then one has $Q^{*}\left(X_{1} \times \cdots \times X_{n}\right)=1$.

Demonstration. If

$$
B \in \Re\left(S_{1} \times \cdots \times S_{n}\right) \text { and } B \subset\left(S_{1} \times \cdots \times S_{n}\right) \backslash\left(X_{1} \times \cdots \times X_{n}\right) \text {, }
$$

then $B$ is contained in a countable union of 1-slices of $S_{1} \times \cdots \times S_{n}$ over points in $S_{j} \backslash X, j=1, \ldots, n$, and so $Q(B)=0$.

Proposition 8. If $X_{1}, \ldots, X_{n}(n \geq 2)$ are jointly Borel-dense of order $n-1$ in $S_{1}, \ldots, S_{n}$, then $X_{1}, \ldots, X_{n}$ is jointly Borel-dense of order $n$ in $S_{1}, \ldots, S_{n}$ if and only if whenever $P_{1}, \ldots, P_{n}$ are laws on $X_{1}, \ldots, X_{n}$, respectively, and $Q$ is a law on $S_{1} \times \cdots \times S_{n}$ with univariate marginals $\bar{P}_{1}, \ldots, \bar{P}_{n}$, then also $Q^{*}\left(X_{1} \times \cdots \times X_{n}\right)=1$.

Demonstration. One direction follows from the preceding proposition. For the converse, let $B \subset\left(S_{1} \times \cdots \times S_{n}\right) \backslash\left(X_{1} \times \cdots \times X_{n}\right)$ be an uncountable member of $\Re\left(S_{1} \times \cdots S_{n}\right)$ not contained in a countable union of 1-slices of $S_{1} \times \cdots \times S_{n}$ over points in $S_{J} \backslash X_{J}, j=1, \ldots, n$. By Corollary 1 , there is an uncountable subset $B_{0}$ of $B, B_{0} \in \Re\left(S_{1} \times \cdots \times S_{n}\right)$, all of whose 1-sections are countable. Let $Q$ be a continuous law on $S_{1} \times \cdots \times S_{n}$ with $Q\left(B_{0}\right)=1$. The univariate marginals of $Q$ on $S_{1}, \ldots, S_{n}$ are therefore continuous and, by Proposition 6, give full outer measure to $X_{1}, \ldots, X_{n}$; thus these marginals are of the form $\bar{P}_{1}, \ldots, \bar{P}_{n}$ for certain laws $P_{1}, \ldots, P_{n}$ on $X_{1}, \ldots, X_{n}$. But $Q^{*}\left(X_{1} \times \cdots \times X_{n}\right)=0$.

COROllary 5. Let $S_{1}, \ldots, S_{n}(n \geq 2)$ be uncountable standard spaces and let $X_{1} \subset S_{1}, \ldots, X_{n} \subset S_{n}$ be u.m. subsets of these. If $X_{\text {, is Borel-dense in }}$ $S_{\text {, for each }} j=1, \ldots, n$, then $X_{1}, \ldots, X_{n}$ is jointly Borel-dense of order $n$ in $S_{1}, \ldots, S_{n}$.

Proof. By induction on $n$, applying Proposition 8: if $P_{1}, \ldots, P_{n}$ are laws on $X_{1}, \ldots, X_{n}$, and $Q$ is a law on $S_{1} \times \cdots \times S_{n}$ with marginals $\bar{P}_{1}, \ldots, \bar{P}_{n}$, the u.m. property allows one to choose sets $B_{J} \in \Re_{(}\left(S_{J}\right), B_{j} \subset X_{j}$, 
$j=1, \ldots, n$, such that $\bar{P}_{j}\left(B_{j}\right)=1$; then

$$
Q^{*}\left(X_{1} \times \cdots \times X_{n}\right) \geq Q\left(B_{1} \times \cdots \times B_{n}\right)=1 .
$$

Say that a triple $(X, Y, Z)$ of separable spaces has property (V) if whenever there are given laws $P_{1}$ on $X \times Y$ and $P_{2}$ on $Y \times Z$ with a common marginal on $Y$, there is also a law $P$ on $X \times Y \times Z$ with marginals $P_{1}$ and $P_{2}$. A study of property (V) was undertaken in Shortt [15].

Proposition 9. If $S_{1}, S_{2}, S_{3}$ are uncountable standard spaces, and $X_{1} \subset S_{1}, X_{2} \subset S_{2}, X_{3} \subset S_{3}$ are such that $X_{1}, X_{2}, X_{3}$ are jointly Borel-dense (of order 3) in $S_{1}, S_{2}, S_{3}$, then $\left(X_{1}, X_{2}, X_{3}\right)$ has property (V).

Demonstration. Suppose that $P_{12}$ and $P_{23}$ are laws on $X_{1} \times X_{2}$ and $X_{2} \times X_{3}$ with a common marginal on $X_{2}$; then $\bar{P}_{12}$ and $\bar{P}_{23}$ are laws on $S_{1} \times S_{2}$ and $S_{2} \times S_{3}$ with the common marginal $\bar{P}_{2}$ on $S_{2}$. Using Lemma 7 of Shortt [15], we see that there is a law $Q$ on $S_{1} \times S_{2} \times S_{3}$ with marginals $\bar{P}_{12}$ on $S_{1} \times S_{2}$ and $\bar{P}_{23}$ on $S_{2} \times S_{3}$. If the marginals of $P_{12}$ and $P_{23}$ on $X_{1}$, $X_{2}, X_{3}$ are $P_{1}, P_{2}, P_{3}$, then the univariate marginals of $Q$ are $\bar{P}_{1}, \bar{P}_{2}, \bar{P}_{3}$. Proposition 7 implies that $Q^{*}\left(X_{1} \times X_{2} \times X_{3}\right)=1$; then $P=Q^{*}$ is a law on $X_{1} \times X_{2} \times X_{3}$ with marginals $P_{12}$ and $P_{23}$.

Proposition 10. If $X_{1}, X_{2}, X_{3}$ are jointly Borel-dense of order 2 in $S_{1}$, $S_{2}, S_{3}$, then $\left(X_{1}, X_{2}, X_{3}\right)$ has property (V) if and only if $X_{1}, X_{2}, X_{3}$ are jointly Borel-dense of order 3 in $S_{1}, S_{2}, S_{3}$.

Demonstration. One direction is established by Proposition 9. For the converse, suppose that $X_{1}, X_{2}, X_{3}$ are jointly Borel-dense of order 2, but not of order 3; Corollary 2 then applies to produce an uncountable set $B$ in $\Re\left(S_{1} \times S_{2} \times S_{3}\right)$ with $B \subset\left(S_{1} \times S_{2} \times S_{3}\right) \backslash\left(X_{1} \times X_{2} \times X_{3}\right)$ and such that each non-empty 1-section of $B$ is a singleton set.

Let $Q$ be a continuous law on $B$; then the univariate marginals of $Q$ on $S_{1}, S_{2}$ and $S_{3}$ are continuous and so give full outer measure to $X_{1}, X_{2}$ and $X_{3}$; thus these marginals are of the form $\bar{P}_{1}, \bar{P}_{2}, \bar{P}_{3}$ for certain laws $P_{1}$, $P_{2}, P_{3}$ on $X_{1}, X_{2}, X_{3}$. Proposition 7 with $n=2$ implies that the bivariate marginals of $Q$ on $S_{1} \times S_{2}$ and $S_{2} \times S_{3}$ give full outer measure to $X_{1} \times X_{2}$ and $X_{2} \times X_{3}$; thus these marginals are of the form $\bar{P}_{12}$ and $\bar{P}_{23}$ for laws $P_{12}$ on $X_{1} \times X_{2}$ and $P_{23}$ on $X_{2} \times X_{3}$.

We claim that there is no law $P$ on $X_{1} \times X_{2} \times X_{3}$ with marginals $P_{12}$ and $P_{23}$ : suppose, however, that it were so. Let $B_{12}$ and $B_{23}$ be the 
projections of $B$ onto $S_{1} \times S_{2}$ and $S_{2} \times S_{3}$, respectively; since one-one measurable images of standard spaces are standard, $B_{12} \in \mathscr{B}_{3}\left(S_{1} \times S_{2}\right)$ and $B_{23} \in \mathscr{B}\left(S_{2} \times S_{3}\right)$. Then $\bar{P}$ (the law induced by $P$ on $S_{1} \times S_{2} \times S_{3}$ ) has marginals $\bar{P}_{12}$ on $S_{1} \times S_{2}$ and $\bar{P}_{23}$ on $S_{2} \times S_{3}$; this forces $\bar{P}\left(B_{12} \times S_{3}\right)$ $=\bar{P}_{12}\left(B_{12}\right)=1$ and $\bar{P}\left(S_{1} \times B_{23}\right)=\bar{P}_{23}\left(B_{23}\right)=1$; but

$$
\left(B_{12} \times S_{3}\right) \cap\left(S_{1} \times B_{23}\right)=B \subset\left(S_{1} \times S_{2} \times S_{3}\right) \backslash\left(X_{1} \times X_{2} \times X_{3}\right),
$$

so that $\bar{P}^{*}\left(X_{1} \times X_{2} \times X_{3}\right)=0$, a contradiction.

COROLlaRY 6. There is an uncountable non-u.m. space $X$ such that $(X, X, X)$ has property $(\mathrm{V})$.

Proof. Let $X$ be as in Proposition 3 with $n=3$ (3rd-order but not 4th-order density); by Corollary 5, $X$ cannot be u.m., but Proposition 9 ensures that $(X, X, X)$ has property $(\mathrm{V})$.

This result should be compared with Theorem 5 of Shortt [15], where it is shown that $(X, Y, Z)$ has property (V) for all separable $Y$ and $Z$ if and only if $X$ is u.m..

Proposition 11. Let $X_{1}, X_{2}, \ldots$ be subsets of uncountable standard metric spaces $S_{1}, S_{2}, \ldots$; then either of (a) condition $\left(\mathrm{K}_{\infty}^{\prime}\right)$ or (b) condition $\left(\mathrm{K}_{\infty}\right)$ together with Martin's Axiom implies the following:

(*) if $P_{1}, P_{2}, \ldots$ are laws on $X_{1}, X_{2}, \ldots$, respectively, and $Q$ is a law on $S_{1} \times S_{2} \times \cdots$ with univariate marginals $\bar{P}_{1}, \bar{P}_{2}, \ldots$, then

$$
Q^{*}\left(X_{1} \times X_{2} \times \cdots\right)=1 \text {. }
$$

If the laws $P_{1}, P_{2}, \ldots$ in $(*)$ are further assumed to be continuous, then either of (a) condition $\left(\mathrm{KK}_{\infty}^{\prime}\right)$ or $(\mathrm{b})$ condition $\left(\mathrm{KK}_{\infty}\right)$ together with Martin's Axiom implies (*).

Demonstration. We use the fact that all laws on $S_{1} \times S_{2} \times \cdots$ are tight. If $K$ is a compact subset of $\left(S_{1} \times S_{2} \times \cdots\right) \backslash\left(X_{1} \times X_{2} \times \cdots\right)$, then, depending on which conditions are assumed, either

(al) $\left(\mathbf{K}_{\infty}^{\prime}\right)$ implies that $K$ is contained in a countable union of 1-slices of $S_{1} \times S_{2} \times \cdots$ over points in $S_{j} \backslash X_{j}, j=1,2, \ldots$, and so $Q(K)=0$, or

(bl) $\left(\mathrm{K}_{\infty}\right)$ implies that $K$ is contained in the union of sets $S_{1} \times S_{2}$ $\times \cdots \times A_{j} \times \cdots, j=1,2, \ldots$, where $A_{j}$ is a subset of $S_{j} \backslash X_{j}$ of cardinality less than $c$. Martin's Axiom (see e.g. Rudin [13,p. 498]) implies that $\widetilde{P_{j}^{*}}\left(A_{j}\right)=0$, so that $Q(K)=0$, or 
(a2) $\left(\mathrm{KK}_{\infty}^{\prime}\right)$ implies that $K$ is contained in a countable union of 1-slices of $S_{1} \times S_{2} \times \cdots$; the continuity of the $\bar{P}_{j}$ implies that $Q(K)=0$, or

(b2) $\left(\mathrm{KK}_{\infty}\right)$ implies that $K$ is contained in a union of sets $S_{1} \times S_{2}$ $\times \cdots \times A_{j} \times \cdots, j=1,2, \ldots$, where each $A_{j}$ is of cardinality less than $c$. Martin's Axiom, together with the continuity of the $\bar{P}_{j}$, implies $\bar{P}_{j}^{*}\left(A_{j}\right)=0$, so that $Q(K)=0$.

In analogy with Proposition 8, it may be that in the presence of the joint Borel-density of $X_{1}, X_{2}, \ldots$ in $S_{1}, S_{2}, \ldots$ of every finite order, condition $(*)$ is necessary and sufficient for some density of order $\infty$, but I have no proof.

Say that a sequence $X_{1}, X_{2}, \ldots$ of separable spaces has the continuous Kolmogoroff property (it is CKP) if whenever a sequence $P_{n}(n=1,2, \ldots)$ of laws on $X_{1} \times \cdots \times X_{n}$ with continuous univariate marginals is given, such that the marginal of $P_{n+1}$ on $X_{1} \times \cdots \times X_{n}$ is $P_{n}$, then there is a law $P$ on $X_{1} \times X_{2} \times \cdots$ having the $P_{n}$ as marginals; if the restriction that the univariate marginals be continuous may be removed, then $X_{1}, X_{2}, \ldots$ has Kolmogoroff's property (it is $\mathrm{KP}$ ). It is rather well known that any sequence of standard or analytic spaces is KP (v. Blackwell [1, Theorem 6]); in fact, only the u.m. property is needed (Shortt [16, Corollary 4.2]).

Proposition 12. Let $S_{1}, S_{2}, \ldots$ be uncountable standard metric spaces and suppose $X_{1} \subset S_{1}, X_{2} \subset S_{2}, \ldots$ are subsets of these;

(1) $\left(\mathrm{K}_{\infty}^{\prime}\right)$ implies $\mathrm{KP}$ for $X_{1}, X_{2}, \ldots$,

(2) $\left(\mathrm{K}_{\infty}\right)$ and Martin's Axiom together imply $\mathrm{KP}$,

(3) $\left(\mathrm{KK}_{\infty}^{\prime}\right)$ implies $\mathrm{CKP}$, and

(4) $\left(\mathrm{KK}_{\infty}\right)$ and Martin's Axiom together imply CKP.

Demonstration. Let $P_{n}$ on $X_{1} \times \cdots \times X_{n}$ be a given consistent sequence of laws; then the induced laws $\bar{P}_{n}$ on $S_{1} \times \cdots \times S_{n}$ are consistent. Therefore, there is a law $Q$ on $S_{1} \times S_{2} \times \cdots$ having the $\bar{P}_{n}$ as marginals. Proposition 11 together with the assumptions in any of (1)-(4) ensures that $Q^{*}\left(X_{1} \times X_{2} \times \cdots\right)=1$. The law $P=Q^{*}$ on $X_{1} \times X_{2} \times \cdots$ has marginals $P_{n}$.

Corollary 7. Assume Martin's Axiom, there is a non-u.m. space $X$ (of cardinality $c$ ) such that $X, X, \ldots$ has the continuous Kolmogoroff property (CKP). (This answers in part a question of D. L. Cohn.) 
Proof. Take $X$ as in Proposition 5 and use Proposition 12 in conjunction with the implication $\left(\mathrm{BB}_{\infty}\right) \Rightarrow\left(\mathrm{KK}_{\infty}\right)$.

The exact relationship between Kolmogoroff's property, infinite-order Borel-density, and, the finite-dimensional marginal problem seems unclear; in particular, I do not know if there are any spaces $X$ such that $X, X, \ldots$ has Kolmogoroff's property, but $(X, X, X)$ does not have property (V).

The Borel-density of products and analytic sets. The following theorem will enable us to obtain some rather surprising results concerning the Borel-density of analytic sets.

Proposition 13. If $X_{1}$ and $X_{2}$ are uncountable separable spaces such that $X_{1} \times X_{2}$ is Borel-dense, then $X_{1}$ and $X_{2}$ are standard.

Demonstration. Consider $X_{1}$ and $X_{2}$ as subsets of the standard spaces $S_{1}$ and $S_{2}$, respectively. If $X_{1} \times X_{2}$ is Borel-dense, there is a standard space $S$ and a Borel isomorphism if: $X_{1} \times X_{2} \rightarrow S$ onto $f\left(X_{1} \times X_{2}\right)$, which is Borel-dense in $S$. Then there is an extension of $f$ to a Borel-isomorphism $\bar{f}$ of $B$ onto $\bar{f}(B)$, where $X_{1} \times X_{2} \subset B \in \Re\left(S_{1} \times S_{2}\right)$, and $f\left(X_{1} \times X_{2}\right) \subset$ $\tilde{f}(B) \in \Re(S)$. Thus $X_{1} \times X_{2}$ is Borel-dense in $B$.

Claim 1. $X_{1}$ and $X_{2}$ are Borel-dense: select any $x_{2} \in X_{2}$; then $X_{1}$ is Borel-dense in $T_{1}=\left\{s \in S_{1}:\left(s, x_{2}\right) \in B\right\}$. Select any $x_{1} \in X_{1}$; then $X_{2}$ is Borel-dense in $T_{2}=\left\{s \in S_{2}:\left(x_{1}, s\right) \in B\right\}$.

Claim 2. $X_{1}$ and $X_{2}$ are analytic (using Lemma 2): $X_{1}=\left\{s \in S_{1}:\right.$ the section of $B$ over the point $s$ is uncountable $\}$, and $X_{2}=\left\{s \in S_{2}\right.$ : the section of $B$ over the point $s$ is uncountable $\}$.

Thus $X_{1}$ and $X_{2}$ are u.m. and Borel-dense in $T_{1}$ and $T_{2}$, respectively; by Corollary $5, X_{1}, X_{2}$ are jointly Borel-dense of order 2 in $T_{1}, T_{2}$. Also, $X_{1} \times X_{2}$ is Borel-dense (of order 1) in $B_{0}=B \cap\left(T_{1} \times T_{2}\right)$.

Thus $\left(T_{1} \times T_{2}\right) \backslash B_{0}$ is contained in a countable union of 1-slices of $T_{1} \times T_{2}$ over points in $T_{1} \backslash X_{1}$ and $T_{2} \backslash X_{2}$. Removing these points from $T_{1}$ and $T_{2}$ leaves uncountable standard spaces $T_{1}^{\prime}$ and $T_{2}^{\prime}$.

Claim 3. $X_{1}=T_{1}^{\prime}$ and $X_{2}=T_{2}^{\prime}$ : if not, say $s \in T_{1}^{\prime} \backslash X_{1}$, then $\{s\} \times T_{2}^{\prime}$ would be an uncountable Borel subset of $B_{0}$ disjoint from $X_{1} \times X_{2}$, a contradiction. 
Now Gödel's Axiom of Constructibility $(V=L)$ implies the existence of totally imperfect uncountable co-analytic sets (Gödel [3]), equivalently, of analytic, non-Borel spaces that are Borel dense. Also, it is known that non-Borel-dense analytic sets exist (in ZFC): see Maitra and Ryll-Nardzewski [9]. For example, so called "universal" analytic sets are of this variety. (For more thoughts along these lines, cf. Grzegorek and RyllNardzewski [4].)

R. Daniel Mauldin [11] has proved that if $A$ is a Borel-dense nonstandard analytic space, then $A$ is not isomorphic with any of its powers $A^{n}, n \geq 2$ or with $A \times[0,1]$; he uses the method of Lusin sieves. We offer an improvement of this result:

COROLlary 7. If $A$ is a Borel-dense analytic non-standard space, then $A$ is not isomorphic with any product $A_{1} \times A_{2}$ of two uncountable (analytic) spaces $A_{1}$ and $A_{2}$.

Proof. From Proposition 13, $A_{1}$ and $A_{2}$ would have to be standard.

COROllary 8. If $A$ is an analytic, non-Borel set Borel-dense in the standard space $S$, then $A \times A$ is not Borel-dense (in any standard space), but $A, A, \ldots$ is jointly Borel-dense of any finite order $n$ in $S, S, \ldots$

Proof. $A$ is u.m., and Corollary 5 applies.

COROLlaRY 9. There are non-u.m. spaces not Borel-dense.

Proof. Let $H$ be a non-u.m. subset of a standard space $S$; then $H \times S$ is not u.m., but also not Borel-dense (from Proposition 13).

Another example may be constructed as follows: let $A$ be an analytic non-Borel-dense space; construct $X \subset A$ such that $X$ and $A \backslash X$ meet every uncountable member of $\Re(A)$. The demonstration is omitted.

Acknowledgement. Thanks go to D. L. Cohn for his careful reading of the manuscript and helpful suggestions.

Added in proof. A. W. Miller has pointed out that in Cohen's original model for the consistency of ZFC $+\left(c=\boldsymbol{\aleph}_{2}\right)$ there are sets $X \subset Y \subset Z$ with $X$ Borel-dense in $Y$ and $Y$ Borel-dense in $Z$, but $X$ not Borel-dense in $Z$. 


\section{REFERENCES}

[1] D. Blackwell, On a class of probability spaces, Proceedings of the 3rd Berkeley Symposium on Mathematical Statistics and Probability, Vol. 2, University of California Press, Berkeley (1956), 1-6.

[2] G. V. Cox, Lusin properties in the product space $S^{n}$, Fund. Math., 108 (1980), 199-120.

[3] K. Gödel, The consistency of the axiom of choice and of the generalized continuum-hypothesis, Proc. Nat. Acad. Sci., 24 (1938), 556-557.

[4] E. Grzegorek and Ryll-Nardzewski, A remark on absolutely measurable sets, Bull. de L'Acad. Polonaise des Sci., (1980), 28 (1980), 229-232.

[5] H. Hahn, Reele Funktionen, (1948), Chelsea.

[6] J. Hoffmann-Jørgensen, The Theory of Analytic Spaces, Various Publications Series No. 10, (1970), Aarhus.

[7] C. Kuratowski, Evaluation de classe Borelien..., Fund. Math., 17 (1933), 249-282.

[8] N. Lusin, Lecons sur les Ensembles Analytiques, Gauthier-Villars, (1930).

[9] A. Maitra and C. Ryll-Nardzewski, On the existence of two analytic non-Borel sets which are not isomorphic, Bull. Acad. Polon Sci., 18 (1970) 177-178.

[10] E. Marczewski (Szpilrajn), The characteristic function of a sequence of sets and some of its applications, Fund. Math., 31 (1938), 207-223.

[11] R. D. Mauldin, On nonisomorphic analytic sets, Proc. Amer. Math. Soc., 58 (1976), 241-244.

[12] S. Mazurkiewicz and W. Sierpinski, Sur une problème des fonctions continue, Fund. Math., 6 (1924), 161-169.

[13] M. E. Rudin, Martin's Axiom in the Handbook of Mathematical Logic, North-Holland Publishing Co., (1977), 491-501.

[14] S. Saks, On the functions of Besicovitch, Fund. Math., 19 (1932), 211-219.

[15] R. M. Shortt, Universally measurable spaces: an invariance theorem and diverse characterisations, Fund. Math., (1983), (to appear).

[16] Strassen's marginal problem in two or more dimensions, Zeitschrift für Warscheinl. 64 (1983), 313-325.

Received May 10, 1982.

Michigan Technological University

Houghton, MI 49931 


\section{PACIFIC JOURNAL OF MATHEMATICS \\ EDITORS}

Donald BABBITT (Managing Editor)

University of California

Los Angeles, CA 90024

Hugo Rossi

University of Utah

Salt Lake City, UT 84112

C. C. Moore and Arthur Ogus

University of California

Berkeley, CA 94720
J. DugunduI

Department of Mathematics

University of Southern California

Los Angeles, CA 90089-1113

R. FinN and H. SAMELSON

Stanford University

Stanford, CA 94305

ASSOCIATE EDITORS

R. ARENS

E. F. BECKENBACH

B. H. NeUMANN

F. WOLF

K. YosHIDA (1906-1982)

\section{SUPPORTING INSTITUTIONS}

UNIVERSITY OF ARIZONA

UNIVERSITY OF BRITISH COLUMBIA

CALIFORNIA INSTITUTE OF TECHNOLOGY

UNIVERSITY OF CALIFORNIA

MONTANA STATE UNIVERSITY

UNIVERSITY OF NEVADA, RENO

NEW MEXICO STATE UNIVERSITY

OREGON STATE UNIVERSITY
UNIVERSITY OF OREGON

UNIVERSITY OF SOUTHERN CALIFORNIA

STANFORD UNIVERSITY

UNIVERSITY OF HAWAII

UNIVERSITY OF TOKYO

UNIVERSITY OF UTAH

WASHINGTON STATE UNIVERSITY

UNIVERSITY OF WASHINGTON 


\section{Pacific Journal of Mathematics}

\section{Vol. 113, No. $1 \quad$ March, 1984}

Flavio E. A. da Silveira, Rational homotopy theory of fibrations $\ldots \ldots \ldots \ldots 1$

Donald M. Davis, Desuspensions of stunted projective spaces ............ 35

Lou van den Dries, Exponential rings, exponential polynomials and

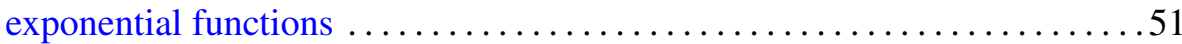

Fred Galvin and Samuel David Shore, Completeness in semimetric

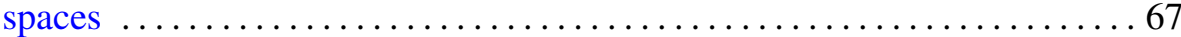

Fereidoun Ghahramani, Compact elements of weighted group algebras . . . 777

Munehiko Itōo, The closed image of a hereditary $M_{1}$-space is $M_{1} \ldots \ldots \ldots 85$

Elvira Laura Livorni, Classification of algebraic surfaces with sectional genus less than or equal to six. I. Rational surfaces .............. 93

H. Alan MacLean, Riesz sets and a theorem of Bochner ............ 115

E. Neher, Jordan triple systems with completely reducible derivation or

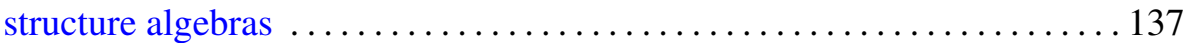

Joe Repka, Shalika's germs for $p$-adic GL( $n)$. I. The leading term $\ldots \ldots \ldots 165$

Joe Repka, Shalika's germs for $p$-adic GL( $n)$. II. The subregular term $\ldots \ldots 173$

Rae Michael Andrew Shortt, Borel density, the marginal problem and isomorphism types of analytic sets $\ldots \ldots \ldots \ldots \ldots \ldots \ldots \ldots \ldots \ldots \ldots \ldots \ldots$

Baruch Solel, The multiplicity functions of invariant subspaces for nonselfadjoint crossed products $\ldots \ldots \ldots \ldots \ldots \ldots \ldots \ldots \ldots \ldots \ldots \ldots . \ldots \ldots$

Su-win Yang, Self-intersection number of immersions and enumeration of nonstable vector bundles

W. M. Zajączkowski, Local solvability of nonstationary leakage problem for ideal incompressible fluid. II 